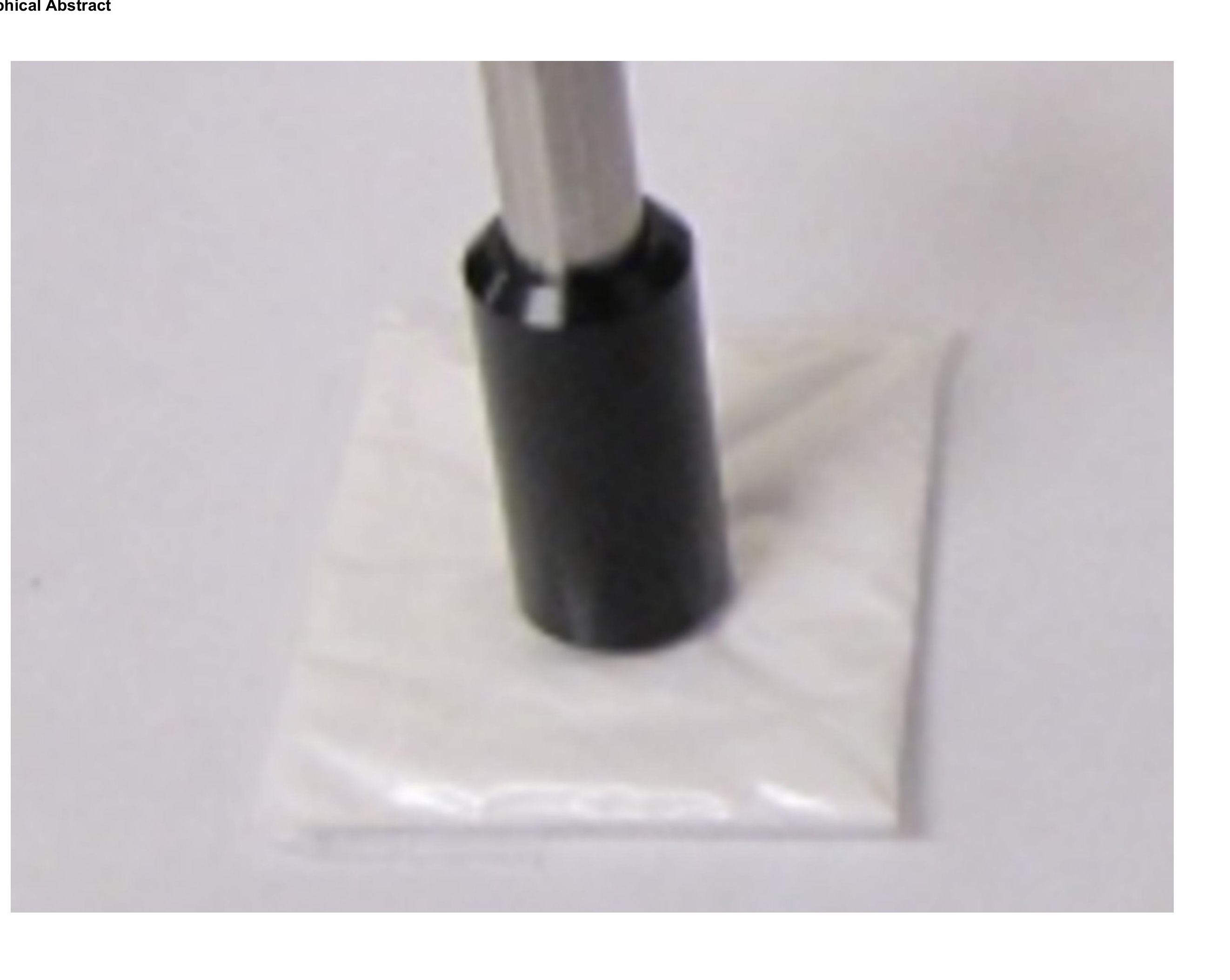

${ }^{*}$ Graphical Abstract

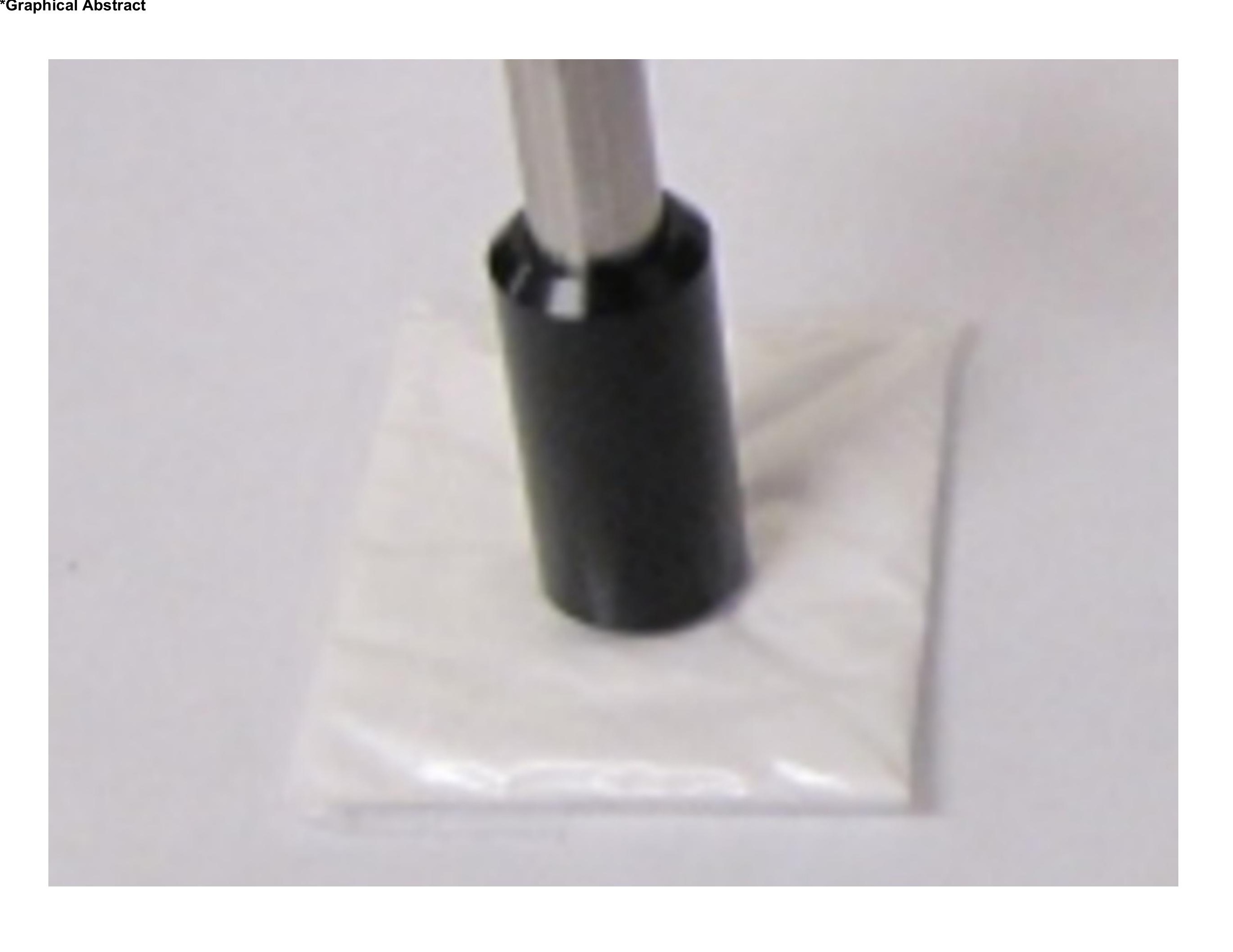




\section{Expanding the Analytical Toolbox for Identity Testing of Pharmaceutical Ingredients: Spectroscopic Screening of Dextrose Using Portable Raman and Near Infrared} Spectrometers

*EMAIL: Jason.Rodriguez@fda.hhs.gov

Division of Pharmaceutical Analysis, Center for Drug Evaluation and Research, US Food and Drug Administration, 645 S. Newstead Ave Saint Louis, MO 63110

CORRESPONDING AUTHOR INFORMATION: Tel (314) 539-3855 Fax (314) 539-2113 


\begin{abstract}
In the pharmaceutical industry, dextrose is used as an active ingredient in parenteral solutions and as an inactive ingredient (excipient) in tablets and capsules. In order to address the need for more sophisticated analytical techniques, we report our efforts to develop enhanced identification methods to screen pharmaceutical ingredients at risk for adulteration or substitution using field-deployable spectroscopic screening. In this paper we report our results for a study designed to evaluate the performance of field-deployable Raman and near infrared (NIR) methods to identify dextrose samples. We report a comparison of the sensitivity of the spectroscopic screening methods against current compendial identification tests that rely largely on a colorimetric assay. Our findings indicate that NIR and Raman spectroscopy are both able to distinguish dextrose by hydration state and from other sugar substitutes with 100\% accuracy for all methods tested including spectral correlation based library methods, principal component analysis and classification methods.
\end{abstract}

Keywords: excipient screening, Raman spectroscopy, near infrared spectroscopy, identification tests, adulteration 


\section{INTRODUCTION}

Dextrose, the common name for D-glucose $\left(\mathrm{C}_{6} \mathrm{H}_{12} \mathrm{O}_{6}\right)$, is a simple sugar usually obtained by the hydrolysis of starch. Dextrose is commonly found as a monohydrate or in its anhydrous form.[1] Dextrose has many uses in food and pharmaceutical industry. In the pharmaceutical industry it is commonly found as both and active ingredient in parenteral solutions and as an excipient (inactive ingredient). [2,3] As an excipient, dextrose has widespread use as a sweetener, reducing agent, bulking agent and soluble carrier for the active pharmaceutical ingredient (API).[4, 5]

Sugars such as dextrose, and the general class of excipients as a whole, have received increased attention due to the increasing complexity arising from globalization of the raw material supply chain. It has become increasingly difficult to assess the quality of purchased raw materials since many ingredients are traded as or derived from commodities and may go through multiple suppliers and dealers before reaching their final destination at the manufacturing facility. These multiple exchanges may increase the potential for economically-motivated adulteration (EMA) of excipients, especially for materials that have acute price fluctuations due to supply shortages.[6] The U.S. FDA has applied a working definition of EMA beginning in 2009 as "the fraudulent, intentional substitution or addition of a substance in a product for the purpose of increasing the apparent value of the product or reducing the cost of its production, i.e., for economic gain."[7] The documented incidences of EMA involve a common vulnerability: Substituted ingredients possessing similar physical and/or chemical properties. These similarities make EMA particularly difficult to detect by conventional tests used by industry. These tests may lack sensitivity and/or specificity.[8-13] High profile cases of EMA of excipients and APIs have been reported over the past ten years which resulted in serious adverse health outcomes. Examples include 46 deaths in Panama in 2006 due to cough and anti-allergy syrup and toothpaste containing diethylene glycol (DEG), imported toothpaste from China containing DEG, 24 children reported dead in Nigeria in 2009 due to paracetamol (acetaminophen) syrup adulterated with DEG,[14] the 2008 heparin crisis due to contamination of raw heparin stock imported from China,[8] and the 2007-2008 melamine contaminations of pet food, milk, and infant formula. 
In 2012, FDA alerted the United States Pharmacopeia (USP) that dextrose excipient was one of 12 excipients considered at elevated risk of adulteration due to an ambiguous identification (ID) test.[14] Manufacturers of finished pharmaceuticals are generally accustomed to relying on a supplier certificate of analysis and performing a compendial ID test in order to release incoming shipments of excipients such as dextrose into their processes. The compendial ID tests for common excipients, many of which involve wet chemical analysis, can be inconclusive with regard to purity and authenticity. Spectroscopic methods are non-destructive, require minimal sample preparation, and can be carried out on portable instruments at the pharmaceutical product manufacturing facility by non-experts. Previous Raman and near infrared studies have indicated that common spectral correlation methods are capable of screening for and detecting adulterant [15] levels which are in the 5-25\% range.[16] The studies presented in this work utilize spectral correlation methods along with multivariate methods to perform enhanced Raman and near infrared (NIR) ID screening of incoming raw ingredients with a particular emphasis on distinguishing between highly similar materials that may have been substituted or mislabeled as authentic dextrose.

Raman and NIR spectroscopies are complementary spectroscopic techniques used to acquire the unique vibrational molecular signature of substances. Both Raman and NIR are commonly available in portable/handheld platforms from a variety of commercial vendors.[17] One of the greatest benefits of using these instruments is their ability to perform rapid interrogation of the sample under study, often in its original packaging, without any additional sample preparation.[16] Raman and NIR instruments can be programmed with methods ranging from correlation-based spectral library tests[18] to more rigorous multivariate-based tests such as principal component analysis (PCA) and soft independent modeling of class analogies (SIMCA).[19, 20] All three approaches were used in this study to evaluate the ability of spectroscopic methods to provide unambiguous ID of dextrose versus monograph methods, dextrose anhydrous versus dextrose monohydrate, dextrose from different manufacturers, and dextrose from the same manufacturers but different production lots.

\section{EXPERIMENTAL METHODS}


Sample Preparation. The dextrose samples as well as other sugars and related excipients used in this work were purchased commercially or taken from an in-house collection of excipients from various manufacturers without further purification. A list of the 32 samples including the manufacturer/source for each is given in Table 1. Approximately 3-4 grams of each sample was transferred to reclosable 2 mil 2x2 inch clear polyethylene bags (Royal Bag, Item: 1004). Screening using Raman and NIR was conducted through the bags as described below.

Compendial Identification Tests. The compendial ID test for dextrose was run on the 32 samples included in the study and was carried out on the exact same samples that were used for the spectral correlation, PCA and SIMCA tests according to the most recent USP monograph method.[1] Briefly, a 1:20 solution of each sample was made by dissolving approximately $0.75 \mathrm{~g}$ of the powder samples taken from the polyethylene bags and mixed with $15 \mathrm{~mL}$ Millipore water followed by shaking to dissolve the solid particles. Five drops of this solution were then added to hot alkaline cupric tartrate in a test tube. The hot alkaline cupric tartrate was made by dissolving approximately $35 \mathrm{~g}$ of cupric sulfate in Millipore water to make $500 \mathrm{~mL}$ and combining in equal volumes with a solution made by dissolving $173 \mathrm{~g}$ of potassium sodium tartrate and $50 \mathrm{~g}$ of sodium hydroxide in Millipore water to make $500 \mathrm{~mL}$. The test tubes containing the test sample solutions and hot alkaline cupric tartrate were heated to approximately $75^{\circ} \mathrm{C}$ in a water bath.

Spectral Collection. Raman spectra in this study were acquired on an EZ-Raman I portable Raman Spectrometer (Enwave Optronics Inc.) using the output of a $785 \mathrm{~nm}$ laser $(340 \mathrm{~mW})$. The TE-cooled $\mathrm{CCD}$ detector was operated at $-50^{\circ} \mathrm{C}$ with variable acquisition times ranging from 1-36 seconds. Acquisition was determined by the software using an algorithm which includes first performing a quick $\sim 1$ second scan on each sample and determining the appropriate integration time to yield a spectrum with $\sim 50,000$ counts. The Raman spectral region analyzed was $300-2000 \mathrm{~cm}^{-1}$. NIR spectra were acquired on a LabSpec 500 portable NIR spectrometer (ASD Inc.). Samples were acquired using the Muglight attachment, which utilizes a tungsten quartz halogen light source. The acquisition time of 0.5 seconds was used for all spectra acquired. The NIR spectral region analyzed was 1100-2100 nm. For 
both Raman and NIR spectra, each sample polyethylene bag was measured 15 times using random sampling at different locations on the polyethylene bag.

Algorithm/Model Construction. Three types of algorithms were used in this work: 1) spectral correlation, 2) PCA and 3) SIMCA. The same spectra were used for all three algorithms. First derivative preprocessing[21] (Gram Polynomial $2^{\text {nd }}$ order 15 point window) was applied to all spectra prior to model development for spectral correlation and PCA. First derivative preprocessing and standard normal variate (SNV) were used for the SIMCA model. First derivative preprocessing was used to reduce the baseline in the Raman and NIR spectra and emphasize the peak information (zero crossings in the first derivative spectra).

Each sample spectrum used for the spectral correlation methods was obtained by taking the average spectrum of the 15 different measurements from random locations on the polyethylene bag described in the previous section. The spectral correlation values reported in this study were calculated using the spectral correlation algorithm and is shown in Equation 1,

$$
S C=\frac{(\text { Library } \cdot \text { Test })^{2}}{(\text { Library } \cdot \text { Library })(\text { Test } \cdot \text { Test })}
$$

where Library indicates the library spectrum and Test indicates an individual spectrum of the material under study. In this study, the Library spectra for dextrose anhydrous and dextrose monohydrate are noted in Table 2. The SC defined in Equation 1 is the square of the spectral covariance between the library and test spectra normalized by the squared norms of the two spectral vectors (i.e., the square of the spectral correlation coefficient). Values for SC range from 1.000, which indicates perfect correlation and 0 , which indicates poor correlation. A 0.95 value threshold is used to determine Pass/Fail samples.

PCA and SIMCA data analysis was performed using PLS Toolbox (Version 7.5.2). The PCA analysis was done on all 15 spectra acquired through the polyethylene bags for each of the 32 samples. Each of the 32 samples was assigned a class. Cross validation (venetian blinds, 6 data splits) was used to help determine the appropriate amount of principal components for each model. The numbers of 
principal components chosen for the model were based on careful comparison between the principal component distributions and examination of the root mean squared error of calibration (RMSEC) trends and root mean squared error of cross validation (RMSECV) values. The SIMCA model was used to perform classification of seven test samples used to challenge the model. These seven samples included one sample each of dextrose anhydrous and dextrose monohydrate as well as samples that erroneously passed the compendial ID test. The SIMCA model contained two classes, one dextrose anhydrous and one dextrose monohydrate class. Each class was comprised of five different samples from five commercial/in-house manufacturers. All 15 spectra acquired through the polyethylene bags were used for each sample, and thus each class contained 75 different spectra for both Raman and NIR. Class predictions were made based on the "strict" criteria. Briefly, each test sample is compared to each of the classes in the SIMCA model and a class assignment was made based on the probability of the sample under study belonging to each of the two classes created. Each of the seven test samples may be assigned to only one class - dextrose anhydrous or dextrose monohydrate. If a sample was found to have low probability of belonging to any of the two classes or found to have high probability of belonging to both the anhydrous or monohydrate classes then no class designation is made and it is designated unclassified. The probability values were calculated and decisions were made by the PLS Toolbox program and are detailed elsewhere.[22] Classification decisions were made using the "combined" decision rule based on the $\mathrm{Q}$ and $\mathrm{T}^{2}$ outlier statistics for each validation sample compared to $\mathrm{Q}$ and $\mathrm{T}^{2}$ distributions for each of the two classes.

\section{RESULTS AND DISCUSSION}

The results of USP-NF compendial ID tests for dextrose applied to all samples included in this study are listed in Table 1. Two examples for typical results of the compendial ID test for dextrose are shown in Figure 1. The compendial ID test defines acceptance criteria as "a copious red precipitate of cuprous oxide is formed."[1] Figure 1A shows a 'Pass' designation while figure 1B shows a 'Fail' designation. The figure shows a clear difference between a precipitate forming and no forming of a precipitate. All samples labeled dextrose (anhydrous and monohydrate), passed the compendial test as 
indicated in Table 1. The test did not have a discernable difference in precipitate formation depending on the form of dextrose-anhydrous or monohydrate. Other substances that may be substituted for dextrose in food or pharmaceuticals such as dextrin, sugar alcohols including mannitol and sorbitol, and table sugar, sucrose, clearly failed the compendial ID test. However there were several non-dextrose samples that erroneously passed the compendial ID test including maltodextrin and maltodextrin/dextrose anhydrous mixtures. Maltodextrins are substances that contain variable length polymers of dextrose ranging from three to 19 units.[23] Other samples which erroneously passed the compendial ID test include lactose monohydrate, galactose anhydrous, and fructose-all reducing sugars While the compendial ID test generally did an adequate job of differentiating dextrose from complex dextrose polymers (cellulose), reduced forms of dextrose (sorbitol) and sucrose (disaccharide of dextrose and fructose), it was not able to adequately distinguish between simpler polymers of dextrose (e.g., maltodextrin), the disaccharide lactose, and the simple sugars fructose and galactose, showing that these sugars may erroneously pass the existing dextrose identification test. Based solely on visual results, it is apparent that the compendial ID test lacks specificity as several different sugars were able to pass as dextrose. Other problems encountered were rooted in the inherent ambiguity of the ID test and analysis of its results. The monograph test calls for adding "a few" drops of the sample solution into "hot" alkaline cupric tartrate which, if dextrose is present, will result in a "copious" red precipitate of cuprous oxide.[1] The subjective nature of these adjectives leaves room for interpretation into how to proceed with the test. One noteworthy ambiguity, for example, is the temperature of "hot" cupric tartrate. Our experience was that the temperature needed was $70-75^{\circ} \mathrm{C}$. Several attempts to use the cupric tartrate at lower temperatures produced inconclusive results. Similarly, the length of time required for precipitate formation is unclear and the exact tone of the "copious" red precipitate is open to interpretation. Beyond the ambiguity issues, the ID test is unable to distinguish between the most fundamental difference in dextrose - whether it is the monohydrate or anhydrous form - and no discernable differences were noted between different manufacturers, grades, lots for the dextrose samples studied. 
Each of the 32 samples was also analyzed using portable Raman and NIR spectrometers to gauge the performance of spectral library-based ID methods. These methods eliminate the need for subjective analyst decisions based on color and precipitate formation and make determinations by the SC value calculated by Equation 1 using a 0.95 threshold for passing samples. The SC values are listed in Table 2. Each sample was run against dextrose monohydrate and dextrose anhydrous library samples, respectively. For all samples studied only the dextrose monohydrate samples pass when compared to the dextrose monohydrate library spectrum and the same is true for anhydrous samples. The related excipients including the five samples that erroneously passed the compendial ID testmaltodextrin/dextrose anhydrous, maltodextrin, lactose monohydrate, fructose and galactose anhydrous - all fail the SC tests for both dextrose monohydrate and dextrose anhydrous. The Raman and NIR spectra for these five samples along and a few related excipients are shown in Figure 2 alongside the library spectra for dextrose monohydrate and dextrose anhydrous. For both Raman and NIR, the Figure 2 spectra and the associated SC values given in Table 2 clearly show that spectral correlation can distinguish between the monohydrate and anhydrous forms. The Raman SC value of the monohydrate against the anhydrous form of dextrose is below 0.07 and the value is below 0.50 when comparing the NIR spectra for each. Both of these values are well below the 0.95 threshold and would be screened as different should a container of the monohydrate form be mislabeled as the anhydrous form and vice versa. The Raman spectra of the monohydrate and anhydrous forms shown in Figure 2A exhibit quite a bit of differences in the overall peak profile throughout the spectral range analyzed. The NIR signatures of the monohydrate and anhydrous pair, shown in Figure $2 \mathrm{~B}$, exhibit quite a bit of similarities compared to the Raman data, but are clearly different in the region above $1700 \mathrm{~nm}$. All other samples have SC values well before the 0.95 threshold as listed in Table 2 and would clearly be flagged as "Fail" when compared with the reference signature of the monohydrate or anhydrous form. The five samples in the sample set that erroneously passed the compendial ID testmaltodextrin/dextrose anhydrous, maltodextrin, lactose monohydrate, fructose and galactose anhydrous 
are clearly distinguished by spectral correlation for both Raman and NIR and possess vastly different Raman and NIR signatures compared to dextrose monohydrate and dextrose anhydrous.

Based on the results of the test set, it is clear that $\mathrm{SC}$ methods can be used to distinguish between the monohydrate and anhydrous form as well as possible substitution-both intentional and accidental — of other sugars and related excipients from dextrose. We also attempted to study whether SC-based methods could be used to screen for different manufacturers, grades, and lots of dextrose. This level of screening is important in achieving the ultimate goal of full traceability of the supply chain. Such analytical techniques would allow the final user to compare the incoming lot at the manufacturing facility to the original spectral signature at initial manufacture. This would lessen the likelihood of EMA or intentional or accidental substitution and could prove especially useful in authenticating incoming ingredients at the final destination. Such authentication of incoming ingredients is important for excipients that are sold or traded as commodities. For such cases multiple transactions between trading partners is common and the process often includes repackaging and relabeling. The Raman first derivative spectra for a collection of commercially available dextrose samples from different manufacturers, grades, and lots are shown in Figure 3 and the first derivative NIR spectra are shown in Figure 4. The average SC values for each set are given and are calculated by taking the average SC value for each spectrum in the two sets—-seven dextrose anhydrous samples and four dextrose monohydrate samples - compared to each other. All seven Raman signatures for the for dextrose anhydrous samples shown in Figure 3A have essentially the same Raman first derivative spectrum and the average SC value for the anhydrous set is 0.999 . The dextrose monohydrate set shows essentially the same trends and the average SC for the monohydrate set is also 0.999 . The NIR first derivative spectra for the two sets shown in Figure 4A and 4B for the anhydrous and monohydrate form of dextrose, respectively, shows similar trends as those observed for the Raman data. The average SC values are 0.987 and 0.999 for the anhydrous and monohydrate forms, respectively. Although unable to provide the sensitivity to discriminate between these sample attributes, the data indicates that to build a successful SC-based library method there really only needs to be one reference spectrum each for the 
anhydrous and monohydrate forms of dextrose and there is no need to build a library with every possible dextrose manufacturer, grade, or lot. While there are certainly exceptions and these should be treated on a case by case basis our SC study indicates that with two reference spectra the identity of a dextrose sample can be determined regardless of manufacturer, grade, or lot. Furthermore SC tests possess the specificity to distinguish between the vibrational signature of dextrose and other sugars and related substances.

The same two groups of commercially available dextrose samples, seven for anhydrous and four for monohydrate, were further studied using PCA methods to study whether multivariate algorithms can be used to separate between different manufacturers, grades, and lots. Figure 5 shows a PCA plot comparing dextrose samples by manufacturer using the Raman data and Figure 6 shows the results using NIR data. Each group of 15 spectra for each sample was designated as a separate class to create a PC model. Each class shown in Figures 5 and 6 are surrounded by a 95\% confidence ellipsoid. As shown in Figure 5A, separation of class based on manufacturer could not be observed with Raman. While both samples from Fisher Scientific Education comingled with each other as would be expected, no conclusions can be drawn regarding the ability of Raman data in a PCA model to distinguish dextrose samples by manufacturer, grade, or lots. Figure 5B, however, shows that PCA models combined with Raman spectroscopy are able to distinguish dextrose monohydrate samples by manufacturer but not by lot. This is indicated by the clear separation of classes based on manufacturer but the comingling between the classes of Fisher Scientific of different lots.

Figure 6 shows a more robust scenario where NIR based PCA models can distinguish between manufacturers for anhydrous dextrose samples (Figure 6A) and can distinguish between manufacturer and lot for dextrose monohydrate samples (Figure 6B). These results are significant because they show the improvement in sensitivity for the spectroscopic technique when using the PCA algorithm instead of the SC library test. The same data was used for both SC methods and PCA models but only PCA was able to distinguish between manufacturers and even grades in the case of NIR. It is also clear that 
Raman based PCA models are not as useful and shows that when analyzing samples for higher order differences one spectroscopy may be better suited than other available spectroscopies.

Since the ultimate goal of this study is to determine the effectiveness of Raman and NIR to perform ID screening of dextrose samples and possible substitutes, we developed a SIMCA model for both Raman and NIR. The SIMCA model was used to make classification decisions and was comprised of two classes: dextrose anhydrous and dextrose monohydrate. Each class included five authentic dextrose anhydrous and dextrose monohydrate samples. As can easily be seen in Figure 7, both Raman and NIR classes for authentic dextrose are clearly distinguishable. An extra principal component is needed to adequately model the NIR data. Compared to Raman, the two NIR classes are more diffuse since NIR is better able to distinguish between dextrose from different manufacturers as has been described above. The results of the seven test samples are shown in Table 3. Three different spectra were analyzed for each of the seven samples. The Raman and NIR SIMCA model was able to correctly identify $100 \%$ of the 21 spectra. The SIMCA results for Raman and NIR clearly indicate that SIMCA can be used to adequately screen out possible dextrose substitutes using both Raman and NIR data.

\section{CONCLUSIONS}

Compendial ID tests were able to distinguish dextrose from some other sugars but failed to discern between dextrose and maltodextrins, dextrose and common reducing sugars, different hydration states of dextrose, and different dextrose grades. The results show that for all 32 samples of dextrose and similar sugars tested passing sample identification was made for all hydration states of dextrose and approximately $20 \%$ of samples that were not dextrose. This indicates the current compendial identification test can result in false positives for sugars similar to dextrose. The compendial ID test was also unable to distinguish between anhydrous and monohydrate form and was unable to distinguish between grades. Such tests are "non-specific" in that they might not be capable of ruling out samples of substances with properties similar to dextrose. The subjective nature of the test based on appearance and lack of control on a critical variable, temperature, leave ample room for interpretation depending on the user and thus raise the possibility of obtaining a false positive for a sample containing dextrose (such as 
a maltodextrin mixture) or a sample containing another reducing sugar. Indeed the current compendial identification test seems to be a variation of Benedict's reagent test which originated in 1909.[24]

Spectroscopic methods are a better choice for identification of dextrose for various reasons including reduced sample preparation which allows for field use, being able to differentiate by hydration state, and being non-destructive towards the sample. Using library-based SC methods, both Raman and NIR were able to distinguish dextrose both from other substances and simple sugars as well as by dextrose hydration state using the 0.95 correlation threshold. The results of this study indicate that a robust library for dextrose screening can be attained with as little as two entries—one for the anhydrous and one for monohydrate forms. This may or may not be the case when looking at other excipients, so each spectral library application should built an update algorithm to decide when additional entries are needed. Some naturally-derived excipients, for example, may show more variability in chemical composition than dextrose and thus may justify having additional entries.

Both Raman and NIR PCA results demonstrated superior sensitivity to distinguish dextrose by hydration state and manufacturer relative to SC methods. Raman PCA models can distinguish monohydrate dextrose by manufacturer but not by lot. NIR PCA models can distinguish anhydrous dextrose by manufacturers but not by lot and can distinguish monohydrate dextrose by manufacturer, lot, and grade. The SIMCA models developed were successful in distinguishing between different dextrose and related sugar samples. While the SIMCA results presented here were promising, it is likely that the library-based SC methods would be easier to implement since they are straightforward and do not require any special calibrations to perform at a manufacturing site. Perhaps the best use of a spectral based ID testing scheme is to use a combination of SC-based library tests and PCA/SIMCA with the former being the first step in the ID process and being used to screen out grossly failing samples such as mislabeled or adulterated samples. Following the initial screen by SC-based tests, PCA/SIMCA could then be used to classify the higher order, minor differences in composition such as those arising from EMA, which are beyond the scope of this pilot. The broader implication of this work demonstrates that 
simple, straight forward spectral ID tests can provide robust first-line alternative to existing compendial

ID tests of incoming raw materials without rigorous model development.

\section{ACKNOWLEDGMENT}

This project was supported in part by the CDER Critical Path and Regulatory Science \& Review

Enhancement Programs. This project was supported in part by an appointment (H.K.S) to the Research

Participation Program at the Center for Drug Evaluation and Research administered by the Oak Ridge

Institute for Science and Education through an interagency agreement between the U.S. Department of

Energy and the U.S. Food and Drug Administration.

\section{DISCLAIMER}

This article reflects the views of the authors and should not be construed to represent FDA's views or policies.

\section{REFERENCES}

[1] USP-NF, in, 2015.

[2] J. Guy, Pharmacology for the Prehospital Professional, Jones \& Bartlett, 2012.

[3] S.M. Weinstein, Plumer's Principles and Practice of Intravenous Therapy, Lippincott Williams \& Williams, 2006.

[4] P. Hull, Glucose Syrups: Technology and Applications, John Wiley \& Sons, 2011.

[5] N.A. Armstrong, A. Patel, T.M. Jones, The compressional properties of dextrose monohydrate and anhydrous dextrose of varying water contents, Drug Dev. Ind. Pharm. 12 (1986) 1885-1901.

[6] Guar Gum Shortage, http://www.rx360.org/AlertsNews/Alerts/GuarGumShortageJanuary2012/tabid/259/Default.aspx, 1/14/2015. [7] Volume 74, Number 64, http://www.gpo.gov/fdsys/pkg/FR-2009-04-06/pdf/E9-7843.pdf, 1/14/2015. [8] A.M. Brustkern, L.F. Buhse, M. Nasr, A. Al-Hakim, D.A. Keire, Characterization of Currently Marketed Heparin Products: Reversed-Phase Ion-Pairing Liquid Chromatography Mass Spectrometry of Heparin Digests, Anal. Chem. 82 (2010) 9865-9870.

[9] J. Moore, A. Ganguly, J. Smeller, L. Botros, M. Mossoba, Standardisation of non-targeted screening tools to detect adulterations in skim milk powder using NIR spectroscopy and chemometrics, NIR news 23 (2012) 9-11.

[10] X. Li, S. Arzhantsev, J.F. Kauffman, J.A. Spencer, Detection of diethylene glycol adulteration in propylene glycol--Method validation through a multi-instrument collaborative study, Journal of Pharmaceutical and Biomedical Analysis 54 (2011) 1001-1006. 
[11] A.B. Champagne, K.V. Emmel, Rapid screening test for adulteration in raw materials of dietary supplements, Vib. Spectrosc. 55 (2011) 216-223.

[12] R.M. El-Abassy, P. Donfack, A. Materny, Visible Raman spectroscopy for the discrimination of olive oils from different vegetable oils and the detection of adulteration, Journal of Raman Spectroscopy 40 (2009) 1284-1289.

[13] J.K. Baird, W.W. Smith, A simple colorimetric method for the specific analysis of food-grade galactomannans, Food Hydrocolloids 3 (1989) 413-416.

[14] L.H. Block, in: The USP Excipients Stakeholder Forum, Meeting \#1, U.S. Pharacopeial Convention, 2013.

[15] J.D. Rodriguez, B.J. Westenberger, L.F. Buhse, J.F. Kauffman, Quantitative Evaluation of the Sensitivity of Library-Based Raman Spectral Correlation Methods, Anal. Chem. 83 (2011) 4061-4067. [16] J.D. Rodriguez, C.M. Gryniewicz-Ruzicka, S. Arzhantsev, J.F. Kauffman, L.F. Buhse, Rapid Screening Methods for Pharmaceutical Surveillance, in: Science and the Law: Analytical Data in Support of Regulation in Health, Food, and the Environment, American Chemical Society, 2014, pp. 149-168.

[17] K. Carron, R. Cox, Qualitative analysis and the answer box: a perspective on portable Raman spectroscopy, Anal. Chem. 82 (2010) 3419-3425.

[18] J.D. Rodriguez, B.J. Westenberger, L.F. Buhse, J.F. Kauffman, Standardization of Raman spectra for transfer of spectral libraries across different instruments, Analyst 136 (2011) 4232-4240.

[19] C.M. Gryniewicz-Ruzicka, J.D. Rodriguez, S. Arzhantsev, L.F. Buhse, J.F. Kauffman, Libraries, classifiers, and quantifiers: A comparison of chemometric methods for the analysis of Raman spectra of contaminated pharmaceutical materials, Journal of Pharmaceutical and Biomedical Analysis 61 (2012) 191-198.

[20] C.M. Gryniewicz-Ruzicka, S. Arzhantsev, L.N. Pelster, B.J. Westenberger, L.F. Buhse, J.F. Kauffman, Multivariate Calibration and Instrument Standardization for the Rapid Detection of Diethylene Glycol in Glycerin by Raman Spectroscopy, Applied Spectroscopy 65 (2011) 334-341. [21] J.F. Kauffman, J.D. Rodriguez, L.F. Buhse, Spectral Preprocessing for Raman Library Searching, American Pharmaceutical Review 14 (2011) 34-40. [22]Eigenvector Wiki: Sample Classification Predictions. http://wiki.eigenvector.com/index.php?title=Sample_Classification_Predictions (accessed 03/19/2015). [23] "Other Caloric Sweetners" http://www.sugar.org/other-sweeteners/other-caloric-sweeteners/, $6 / 17 / 2015$.

[24] S.R. Benedict, A Reagent for the Detection of Reducing Sugars, J. Biol. Chem. 5 (1909) 485-487. 
Table 1: Properties and compendial identification test results for dextrose samples and related excipients included in study.

\begin{tabular}{|c|c|c|c|}
\hline Sample & Manufacturer & Grade $^{\mathrm{a}}$ & Compendial ID \\
\hline Dextrose Anhydrous & Sigma Life Science & EP/BP/JP/USP & Pass \\
\hline Dextrose Anhydrous & Acros & Research & Pass \\
\hline Dextrose Anhydrous & Alfa Aesar & Research & Pass \\
\hline Dextrose Anhydrous & Fisher Science Education & Research & Pass \\
\hline Dextrose Anhydrous & Fisher Science Education & Research & Pass \\
\hline Dextrose Anhydrous & Fisher Scientific & EP/BP/USP & Pass \\
\hline Dextrose Anhydrous & Fisher Scientific & Research & Pass \\
\hline Dextrose Anhydrous & Fisher Scientific Education & Research & Pass \\
\hline Dextrose Anhydrous & Macron Fine Chemicals & USP & Pass \\
\hline Dextrose Anhydrous & Sigma & USP & Pass \\
\hline Dextrose Anhydrous & Sigma Life Science & EP/BP/JP/USP & Pass \\
\hline Dextrose Monohydrate & Sigma-Aldrich & USP & Pass \\
\hline Dextrose Monohydrate & Fisher Science Education & Research & Pass \\
\hline Dextrose Monohydrate & Fisher Scientific & USP & Pass \\
\hline Dextrose Monohydrate & Fisher Scientific & USP & Pass \\
\hline Dextrose Monohydrate & Fisher Scientific Education & Research & Pass \\
\hline Dextrose Monohydrate & In-house library & Research & Pass \\
\hline Dextrose Monohydrate & In-house library & USP & Pass \\
\hline Beta Cyclodextrin & In-house library & Research & Fail \\
\hline Dextrin & In-house library & Research & Fail \\
\hline Ethylcellulose & In-house library & Research & Fail \\
\hline Fructose & Fisher Scientific Education & Research & Pass \\
\hline Galactose Anhydrous & Fisher Scientific & Research & Pass \\
\hline Lactose Monohydrate & In-house library & Research & Pass \\
\hline Maltodextrin & In-house library & Research & Pass \\
\hline Maltodextrin/Dextrose Anhydrous & In-house library & Research & Pass \\
\hline Mannitol & In-house library & Research & Fail \\
\hline Microcrystalline Cellulose & In-house library & Research & Fail \\
\hline Partly Pregelatinized Starch & In-house library & Research & Fail \\
\hline Sorbitol & In-house library & Research & Fail \\
\hline Sucrose & Fisher Scientific & $\mathrm{EP} / \mathrm{BP} / \mathrm{NF}$ & Fail \\
\hline Sucrose & Sigma & EP/BP/USP & Fail \\
\hline
\end{tabular}

aAbbreviations: British Pharmacopeia (BP), European Pharmacopeia (EP), Japanese Pharmacopeia (JP), National Formulary (NF) and United States Pharmacopeia (USP) 
Table 2: SC-based ID test results for dextrose samples and related excipients included in study.

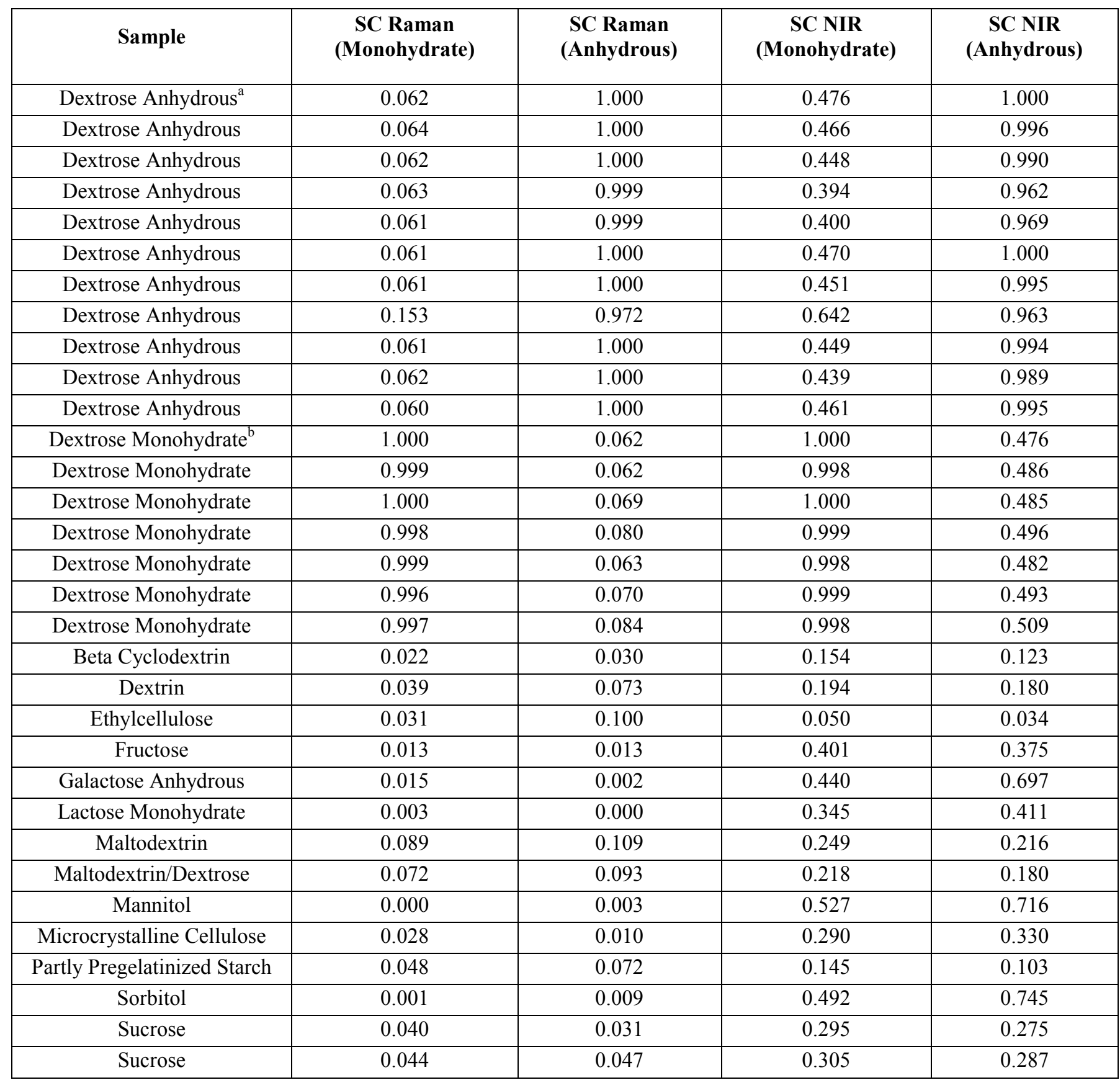

${ }^{a}$ Designated the library reference sample for dextrose anhydrous.

${ }^{\mathrm{b}}$ Designated the library reference sample for dextrose monohydrate. 
Table 3: SIMCA Results for NIR and Raman.

\begin{tabular}{|c|c|c|c|c|c|}
\hline Test Sample & $\begin{array}{c}\text { \# of } \\
\text { Spectra }\end{array}$ & $\begin{array}{c}\text { In Dextrose } \\
\text { Anhydrous Class }\end{array}$ & $\begin{array}{c}\text { In Dextrose } \\
\text { Monohydrate } \\
\text { Class }\end{array}$ & Unclassified & $\begin{array}{c}\text { \% Correct } \\
\text { Classification }\end{array}$ \\
\hline \multicolumn{6}{|c|}{ Raman } \\
\hline Dextrose Monohydrate & 3 & 0 & 3 & 0 & $100 \%$ \\
\hline Dextrose Anhydrous & 3 & 3 & 0 & 0 & $100 \%$ \\
\hline Galactose Anhydrous & 3 & 0 & 0 & 3 & $100 \%$ \\
\hline Lactose Monohydrate & 3 & 0 & 0 & 3 & $100 \%$ \\
\hline Maltodextrin & 3 & 0 & 0 & 3 & $100 \%$ \\
\hline Maltodextrin/Dextrose Anhydrous & 3 & 0 & 0 & 3 & $100 \%$ \\
\hline \multicolumn{6}{|c|}{ NIR } \\
\hline Fructose & 3 & 0 & 0 & 3 & $100 \%$ \\
\hline Lactose Monohydrate & 3 & 0 & 0 & 3 & $100 \%$ \\
\hline Maltodextrin & 3 & 0 & 0 & 3 & $100 \%$ \\
\hline Maltodextrin/Dextrose Anhydrous & 3 & 0 & 0 & 3 & $100 \%$ \\
\hline
\end{tabular}




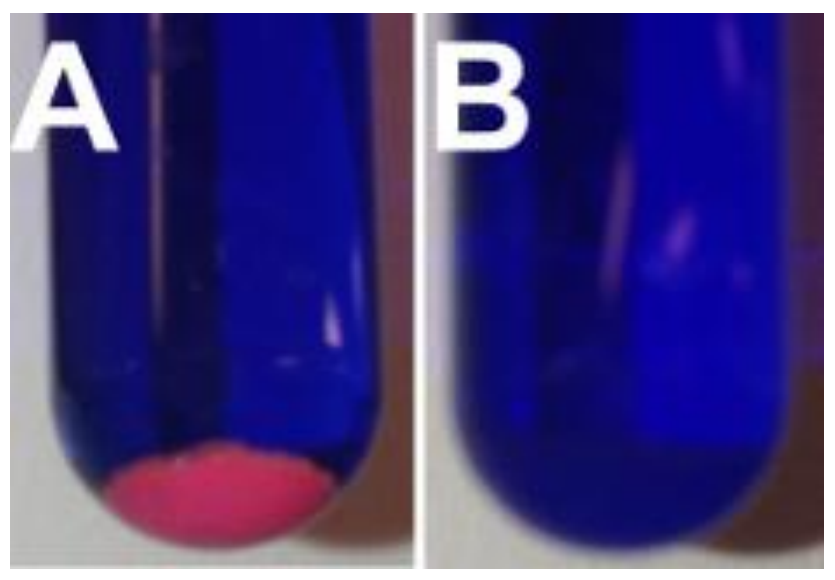

Figure 1. The typical results of the compendial identification test for A) sample that contains dextrose and B) sample that does not contain dextrose. 

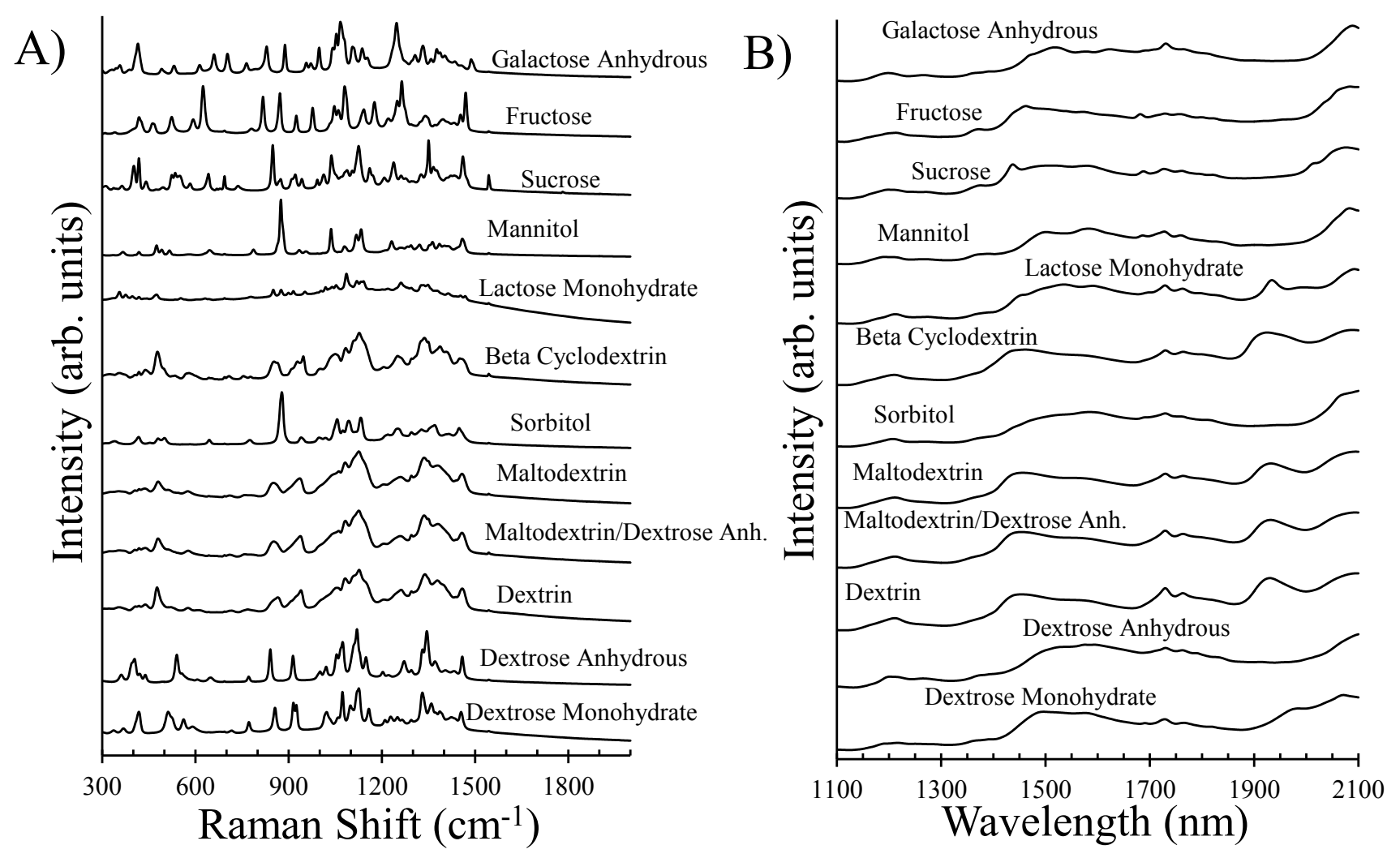

Figure 2. A) Raman and B) NIR spectra for dextrose monohydrate, dextrose anhydrous and other related excipients. 

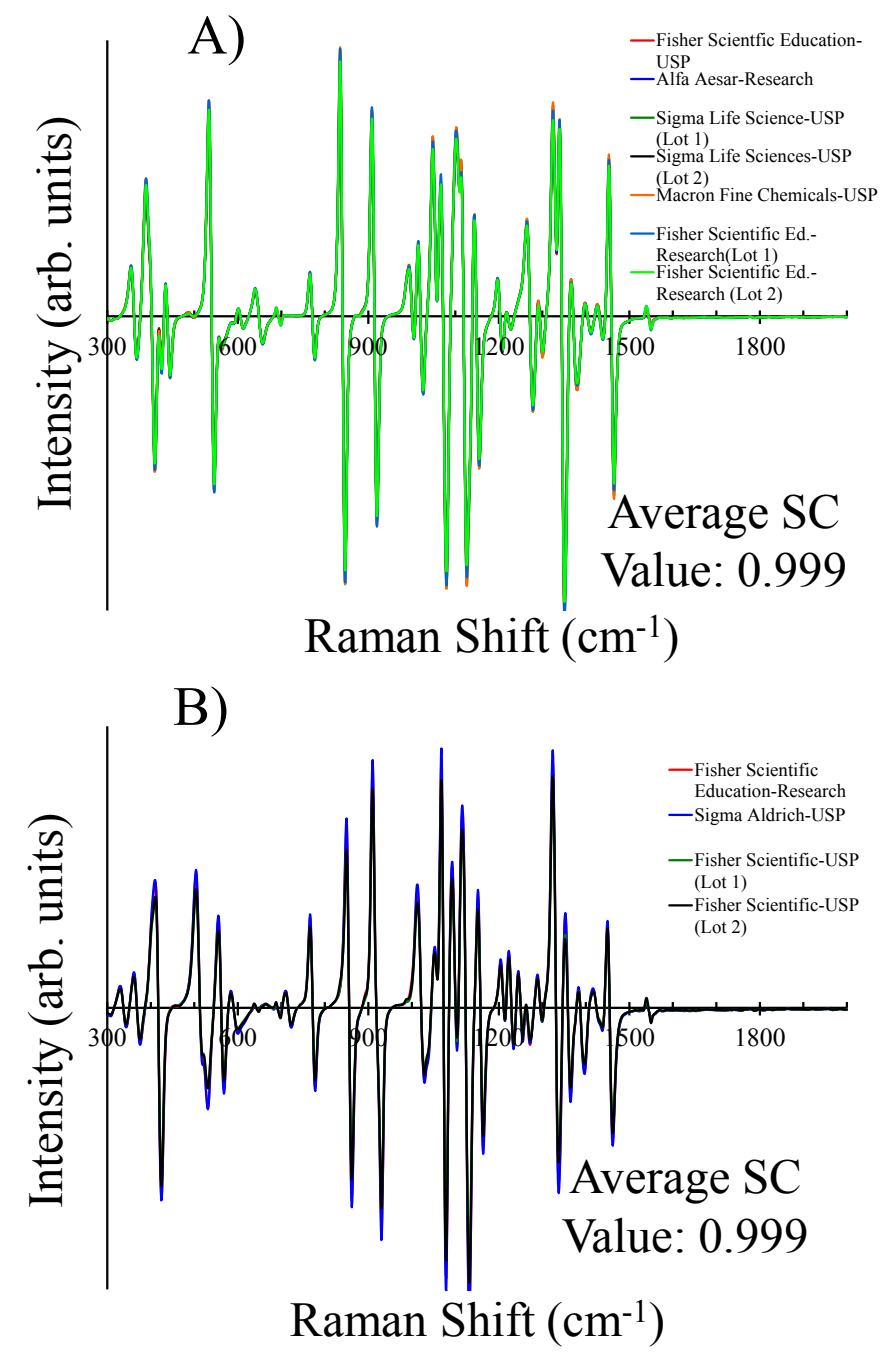

Figure 3. The Raman first derivative spectra ( $2^{\text {nd }}$ order, 15 point window) for A) dextrose anhydrous and B) dextrose monohydrate samples from different manufacturers, grades and lots. 

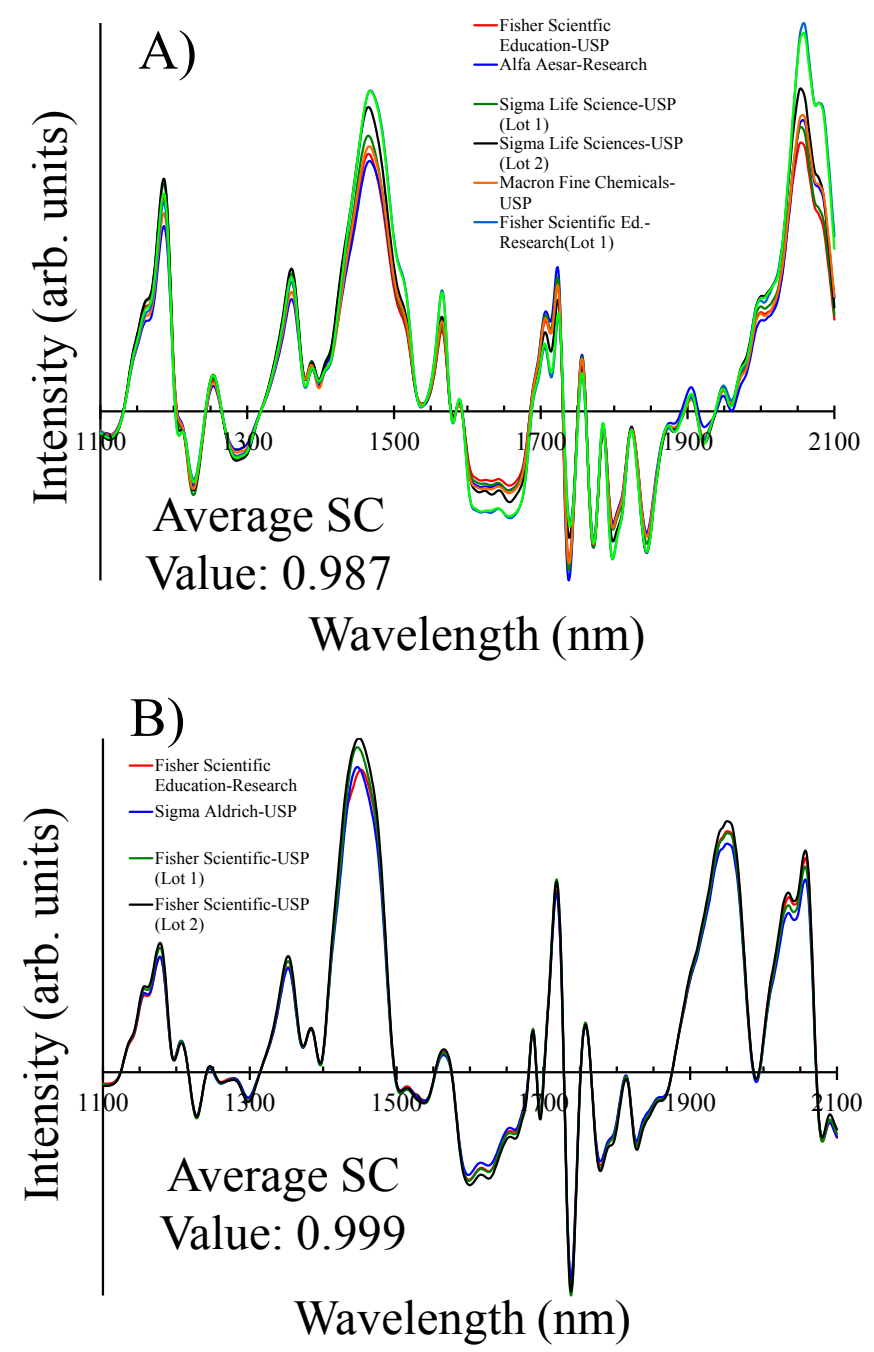

Figure 4. The NIR first derivative spectra ( $2^{\text {nd }}$ order, 15 point window) for A) dextrose anhydrous and B) dextrose monohydrate samples from different manufacturers, grades and lots. 
Figure 5. PCA plots for A) dextrose anhydrous and B) dextrose monohydrate based on the Raman spectra. Three principal components were used for the models. 
Figure 6. PCA plots for A) dextrose anhydrous and B) dextrose monohydrate based on the NIR spectra. Three principal components were used for the models.
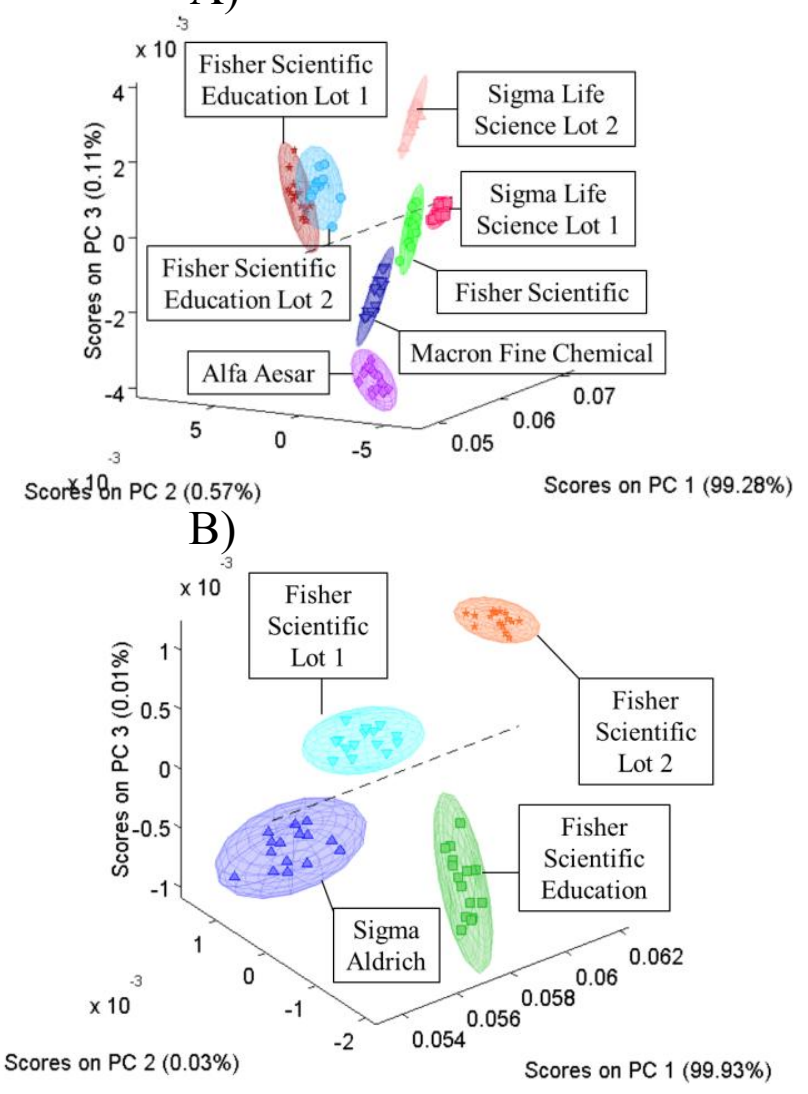


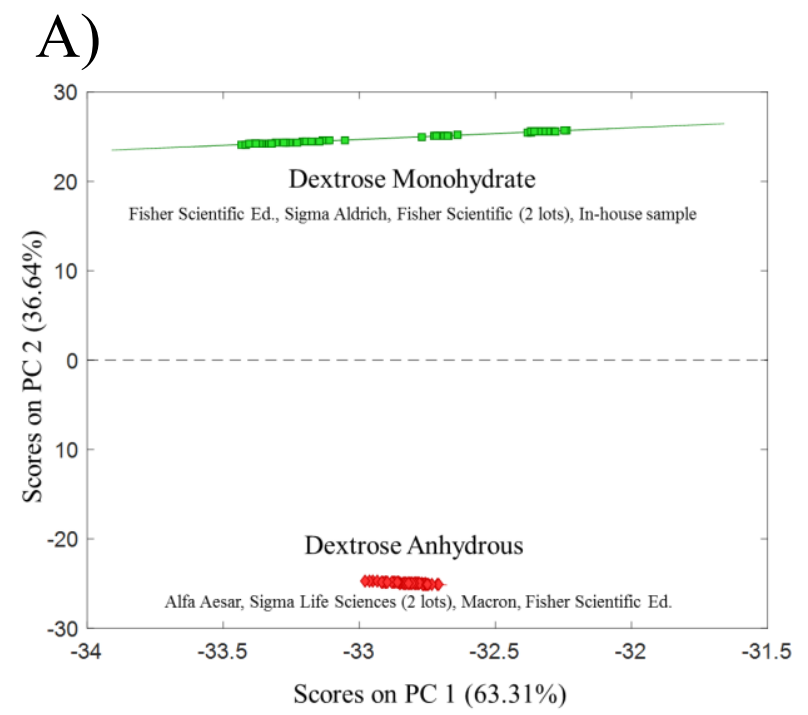

B)

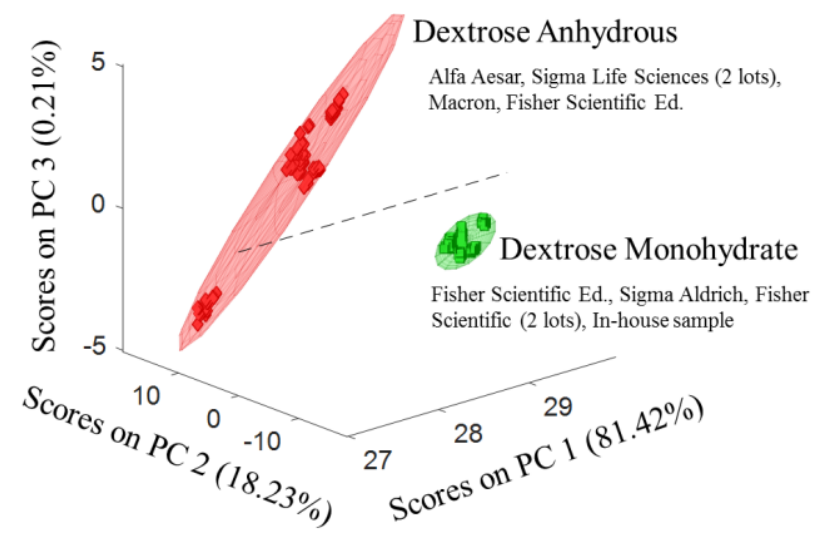

Figure 7. PCA plots for A) Raman and B) NIR classes used for development of the SIMCA model. The SIMCA model contains two classes, each comprised of five different dextrose anhydrous and monohydrate samples. Two principal components were used for each class in the Raman model and three principal components were used for each class in the NIR model 
TOC Graphic

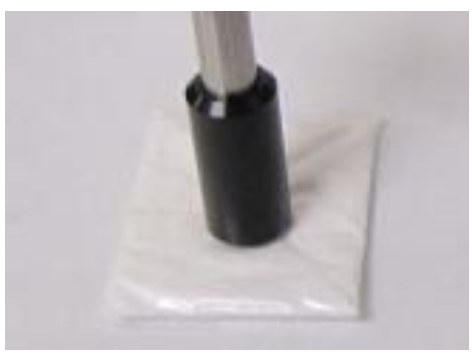

14

15

16

17

18

19

20

21

22

23

24

25

26

27

28

29

30

31

32

33

34

35

36

37

38

39

40

41

42

43

44

45

46

47

48

49

50

51

52

53

54

55

56

57

58

59

60

61

62

63

64

65 